\title{
A Common Beverage with Miraculous Oral Health Benefits - A Review on Green Tea
}

\author{
${ }^{1 *}$ Divya Pandya Bds ${ }^{\text {(Pg student) }}$ \\ ${ }^{I}$ Postgraduate Student, Department Of Oral Medicine and Radiology, \\ Hitkarini Dental College and Hospital, Jabalpur, Madhya Pradesh, India.
}

\begin{abstract}
Nature has bestowed on us a very rich botanical wealth and a large number of assorted types of plants grown in different parts of country. Plants are the richest resource of drugs in traditional systems of medicine. Green tea is one of the most ancient and popular therapeutic beverages consumed around the world. Green tea in its purest form has always influenced human health from generations and day by day scientific evidences throughout the world are making people aware of health benefits associated with this herbal drink. Though green tea is not officially recognized as a medical agent, it is one of the most researched plant-based remedies with possible benefits like promotion of cardio-vascular health, cancer prevention, skin protection, antioxidant activity and many more including improvement of various oral health conditions. This review highlights some recent facts with relevance to current status of green tea benefits in oral health.
\end{abstract}

Keywords: Antioxidant, Green tea, Oral health, Plant products

\section{Introduction}

Before the development of pharmaceutical industry, herbal products were used for curing various conditions. The concept of using natural and herbal products is very old. Humans have used these products for curing various diseases and become popular accordingly. In terms of oral care, most commonly used herbal product is "Miswak" that become popular in several communities as an effective, cheap and efficient way of maintaining oral hygiene. ${ }^{1}$ Since ancient times, plants have been an exemplary source of medicine. Ayurveda and other Indian literature mention the use of plants in treatment of various human ailments. India has about 45,000 plant species and among them, several thousands have been claimed to possess medicinal properties. Traditional system of medicine is found to have utilities as many accounts. Due to population rise adequate supply of drug and high cost of treatment in side effect along with drug resistance has been encountered in synthetic drugs, which has lead to an elevated emphasis for the use of plants to treat human diseases. The affordability of herbals has also drawn the attraction towards their use. India is one of the oldest civilizations which is known for rich repository of medicinal plants. ${ }^{2}$ Similarly, beneficial effects of using various types of tea leaves have been a common topic of discussion in general public and professionals. According to Chinese legend, tea has a known history of over 4000 years ago which was discovered accidentally by an emperor, while according to other sources the Chinese have been drinking tea since 3000 B.C. and over millions of acres are devoted to its cultivation. Currently, it is being used in almost every country around the world and is now been grown in India, Sri Lanka, Iran, Java and Japan. ${ }^{1}$

Green tea (botanical name of tea plant is Camellia Sinensis) belonging to the family Theaceae has been explored in the recent years for its beneficial effects on oral health. It can be available as a shrub or evergreen tree. Its leaves may vary from exstipulate, lanceolate to obovate up to $30 \mathrm{~cm}$ long, $2-5 \mathrm{~cm}$ broad, pubescent, sometimes becoming glabrous, serrate, acute or acuminate. ${ }^{1}$ Tea is the most consumed drink in the world after water. Green tea is a 'non-fermented' tea and contains more catechins than black tea or oolong tea. Catechins are in vitro and in vivo strong antioxidants. In addition, its content of certain minerals and vitamins increases the antioxidant potential of this type of tea. Presently, it is cultivated in at least 30 countries around the world. Tea beverage is an infusion of the dried leaves of Camellia sinesis. It is a widely used medicinal plant by the trials throughout India, China and popular in various indigenous system of medicine like Ayurveda, Unani and Homoeopathy Green tea has been consumed throughout the ages in India, China, Japan and Thailand. ${ }^{2,3}$

\section{Chemical composition of green tea}

One third part of bioactive compounds in green tea is contributed by polyphenols that is the most interesting constituent. The main type of polyphenols are called catechins (flavan-3-ols) also known as tannins serve as astringency constituent. Dr. Michiyo Tsujimura extracted the catechins from tea leaves in 1929 and studied their functions for the first time during his work at The Institute of Physical and Chemical Research, Japan. Most important kind of catechins include epigallocatechin-3-gallate (EGCG; 59\%), epigallocatechin (EGC; 19\%), epicatechin-3-gallate (ECG; 13.6\%) and epicatechin (EC; 6.4\%) including EGCG and EGC are reported for their presence in green tea. Both the types along with ECG exhibit antimicrobial activity. ECG 
reviles the possibility of protection against urinary tract infections (UTI) as it is reported to be excreted by kidney while it is also extracted in bile along with EGCG. Large variations can be observed in the chemical structures of catechins due to dissimilar composition. The chemical composition of green tea also includes: proteins (15-20\% dry weight), whose enzymes constitute an important fraction; amino acids (1-4\% dry weight) such as theanine or 5-N-ethylglutamine, glutamic acid, tryptophan, glycine, serine, aspartic acid, tyrosine, valine, leucine, threonine, arginine, and lysine; carbohydrates (5-7\% dry weight) such as cellulose, pectins, glucose, fructose, and sucrose; minerals and trace elements (5\% dry weight) such as calcium, magnesium, chromium, manganese, iron, copper, zinc, molybdenum, selenium,sodium, phosphorus, cobalt, strontium, nickel potassium, fluorine, and aluminum; and trace amounts of lipids (linoleic and $\alpha$-linolenic acids), sterols (stigmasterol), vitamins (B, C, E), pigments (chlorophyll, carotenoids), and volatile compounds (aldehydes, alcohols, esters, lactones, hydrocarbons). The fresh leaves contain, on average, 3-4\% of alkaloids known as methylxanthines, such as caffeine, theobromine, and theophylline. Green tea also constitutes an important source of antioxidants, such as carotenoids, tocopherols (vitamin $\mathrm{E}$ derivatives) and vitamin $\mathrm{C}$ which is also present in fruits and vegetables. Tea also contains minerals like zinc, selenium and manganese that function as co-factors in antioxidant enzymes. ${ }^{1-9}$

\section{General benefits of green tea}

Numerous therapeutic effects of green tea are reported after human clinical trials which include:

1. Decrease chances of cardiovascular diseases

2. Anti hypertensive

3. Reduces cholesterol

4. Anti bacterial

5. Anti viral

6. UV protector

7. Reduction of obesity

8. Enhancement of bone mineral density

9. Provides neuro- protective power in diseases like Alzheimer's and Parkinson's

10. Treatment of stomach discomfort, indigestion, vomiting, diarrhea and flatulence

11. Enhancement of brain functioning

12. Maintains blood glucose ${ }^{1}$

13. Prevention of breast, prostate, colorectal and pancreatic cancers

14. Beneficial in pregnancy ${ }^{6}$

15. Effective in alleviating symptoms of acne and eczema

16. Good vision

17. Prevents hair loss

18. Effective in renal failure

19. Treatment of rheumatoid and osteoarthritis

20. Antiallergic potentials ${ }^{9}$

21. Anti-aging

22. Boost up immunity

23. Prevention of liver diseases

24. Relives psychological stress

25. Reduces severity of asthma

26. Prevent food poisoning ${ }^{10}$

\section{General mechanism of action of green tea}

The retardation of the growth and development of neoplasms by inhibition of tumor initiation and promotion, induction of apoptosis, and inhibition of cell replication rates is due to the anticarcinogenic properties of green tea polyphenols. The polyphenols antioxidant potential of green tea is directly related to the combination of aromatic rings and hydroxyl groups that make up their structure and is a result of binding and neutralization of free radicals by the hydroxyl groups. In addition, the activity of hepatic detoxification enzymes is stimulated by green tea polyphenols, thereby promoting detoxification of xenobiotic compounds and are also capable of chelating metal ions, such as iron, that can generate radical oxygen species. The production of arachidonic acid metabolites such as pro-inflammatory prostaglandins and leukotrienes is inhibited by green tea polyphenols, which results in a decreased inflammatory response. EGCG has the ability to block inflammatory responses to ultraviolet A and B radiation, as well as, significantly inhibiting neutrophil migration that occurs during the inflammatory process and this has been demonstrated by human and animal studies. There exists a synergistic interaction between green teas caffeine content and catechin polyphenols that can result in prolonged stimulation of thermogenesis. Studies have also shown green tea extracts are capable of reducing fat digestion 
by inhibiting the activity of certain digestive enzymes. Although the exact mechanism is unknown, green tea catechins have been shown to significantly raise levels of Lactobacilli and Bifidobacteria while at the same time decreasing the levels of numerous potential pathogens. Green tea demonstrates antibacterial properties against a variety of gram-positive and gram-negative species. Other than acting as antioxidants, polyphenols have additional mechanisms in which they reduce oxidation level. It binds metal ions such as iron and copper and prevents their participation in oxidation reactions, forming hydroxyl radical. Prevents redox sensitive transcription factors activation that amongst others things serve as mediators of inflammatory reactions and suppress oxidation stimulants such as induced nitric oxide synthase (iNOS), cyclooxygenase 2 (COX-2), lipoxygenase 2 (LOX-2) and xanthine oxidase and Induction of antioxidant enzymes like glutathione Stransferase and super oxide dismutase. ${ }^{3}$

\section{Role of green tea in oral cavity}

Green tea which is essential source for providing polyphenol antioxidants has the ability to protect against various oral diseases such as dental caries, gingivitis, periodontitis, halitosis and oral malignancy (protection and regression). In addition it can prevent from oral oxidative stress, inflammation resulting due to cigarette smoke and reduces dentin erosion and abrasion. ${ }^{1}$

Role as antioxidant - Polyphenols have shown anti-oxidative activity by neutralization of free radicals in the body. They are also known to reduce or prevent their detrimental effects and restrain ROS generation to inhibit the lysosomal secretions. The hydrogen releasing property of catechin and epicatechin results in scavenging effects. Green tea indirectly can inhibit pro oxidant enzymes, redox sensitive transcription factors and induction of antioxidant enzymes. ${ }^{1,3,5,7-10}$

Effects on periodontal and gingival health - The gingival crevice is a physiological zone surrounded by gingiva and tooth margins. A colony of microorganisms resides in this space and most common of that are anaerobes. In pathological conditions, these spaces extend and periodontal pockets filled with serum exudates and large colonies of polymorphs. Additionally, oxidative stress supports the development of diseased conditions. The microbes commonly present in such conditions are Prevotella spp and Porphyromonas gingivalis. ${ }^{1}$ Green tea consumption also reduces alveolar bone loss. EGCG may prevent alveolar bone resorption that occurs in periodontal diseases by inhibiting the expression of MMP-9 in osteoblasts and formation of osteoclast. Oxidative stress plays an important role in the pathogenesis of periodontal disease as well as many other disorders, and it is believed that antioxidants can defend against inflammatory diseases. Daily intake of green tea is significantly associated with bleeding on probing (BOP), probing depth (PD) and clinical attachment loss (CAL). ${ }^{4-6,8,10}$ Gadagi et al conducted an in vivo study on 50 patients with chronic periodontitis out of which 25 had diabetes to analyze the drug release pattern from green tea strips and concluded that local delivery using green tea extract could be used as an adjunct in the treatment of chronic periodontitis in diabetic and non-diabetic individuals. ${ }^{11}$

Use of green tea in halitosis and in mouth wash - The main reagents responsible for halitosis (bad breath) are volatile sulfide compounds such as hydrogen sulfide (H2S), dimethylsulfide [(CH3)2S] and methyl mercaptan $(\mathrm{CH} 3 \mathrm{SH})$. These reagents degrade in the oral cavity by proteolytic reactions primarily by anaerobic gram negative bacteria consuming numerous sulfur-containing substrates including saliva, food debris, epithelial cells and blood. Green tea is well known for antibacterial properties against anaerobic microorganisms. Green tea can abolish bad breath by suppressing anaerobic bacteria and eliminating the production of volatile sulfur compounds. Deodorant action of ingredients decreases in the following order: EGCG > EGC > ECG > EC. The deodorizing action of EGCG is based on a chemical reaction of EGCG and MSH and introducing methylsulfinyl/methylthio group into the B ring of EGCG. During this reaction, a methylthio group is supplemented in orthoquinone form of the catechin produced by oxidation, hence eliminating the halitosis. ${ }^{1,3-5,9}$ Moghbel et al did a comparative study on 25 females with a green tea mouthwash containing $1 \%$ tannin with $10 \%$ ethanol, a alcohol free mouthwash and a green tea herbal mouthwash with $0.2 \%$ chlorhexidine and concluded that herbal green tea mouthwash reduce aerobic mouth bacterial load and prevent plaque formation and over come halitosis and is safe and nontoxic mouthwash especially for children and pregnant women. ${ }^{12}$ Forouzanfar et al did a clinical study to investigate the effect of green tea mouth wash on dental plaque and gingival inflammation following a periodontal surgery and analysed a significant effect of green tea mouth wash on reducing plaque index, gingival index and bleeding on probing and recommended it as a safe, antiinflammatory and anti-microbial mouthwash for gingival inflammation. ${ }^{13}$

Green tea and dental caries - Dental carries is a pathological condition resulting due to demineralization of tooth structure because of bacterial infections and/or deficiency of nutrients. Oral microbes 
are responsible for carries development, out of which mainly streptococcus mutants are most active. The oral antimicrobial peptides are known to reduce the bacterial activity. Green tea leaves are known to be rich in fluoride and other components such as polyphenols (catechins) that play a supportive role in resisting dental carried as reported in many studies. The beneficial role of fluoride is well known as it inhibits the bacterial growth and helps re-mineralization of dental tissues. Cariogenic bacteria release glucans with the help of glucosyltransferase (GTase) which are branched and facilitate adherence of microbes to tooth surface. . $^{1,3-7,7,10,14,15}$

Anti-carcinogenic and reduces cigarette smoke induced inflammation - Smoking affects the homeostasis and injurious to oral health. The effects of smoking range from simple mucosal erythema to premalignant conditions and oral cancer. Smoking intensifies oral malignancies in response to oral inflammatory diseases due to compromised status of salivary antioxidants. Diminished activity of numerous salivary enzymes is observed during smoking hence compromising the protection against oxidative damage. In addition, smoke coming from tobacco comprised of reactive oxygen species (ROS) including superoxides, hydroxyl radical/hydrogen peroxide. The toxicity is further added due to the formation of nitric oxide (NO). Superoxide reacts chemically with $\mathrm{NO}$ to form peroxynitrite (ONOO). The inflammatory transcription factor pathway (NFkB) is triggered by ONOO due to activation of IkB kinase (IKK). The cascade of changes results in the intensification of expressions, NOS activity and chronic inflammation in tissues even by exposing to a very minute dose of smoke. Green tea can be beneficial for cigarette smokers. Catechins present in green tea are capable of scavenging superoxide oxide, NO, and ONOO. In addition, EGCG has the ability to suppress activation of NF-kB that leads to inhibition of phosphorylation. It can further trigger the collapse of the inhibitory sections IkB-a in human pulp cells. The IkBa is also accountable for overturning nuclear transfer of NF-kB operating subunits (p65 and p50) and stimulation of pro-inflammatory genes. EGCG resulted in decreased NF-kB expression and reduction of proteins facilitated including matrix metalloproteinase-9 (MMP9). The MMP-9 is implicated in the breakdown of extracellular matrix, nterleukin-8 (IL-8) and NOS present in bronchial epithelium. There are more than 4700 oxygen/nitrogen species and reactive chemical compounds in the cigarette smoke. Nicotine is the main chemical compound released from cigarettes that affects gingival and periodontal health. Nicotine stimulates apoptosis across ROS generations of human gingival fibroblasts (HuGF). The metabolites of nicotine such as tobaccospecific nitrosamines (TSNAs) are considered major carcinogens. Another smoke compound (acrolein) is a risk factor for developing periodontal diseases and toxic to HuGF. It inhibits normal functioning of gingival fibroblasts due to interruption with cells attachment and proliferation. In advance stages, acrolein can prompt inflammatory conditions and cancer onset. Green tea extract and EGCG can extinguish acrolein and other unsaturated aldehydes, hence reducing acrolein toxicity. Green tea consumption can diminish oxidative and inflammatory tissue injuries in the oral cavity caused by cigarette smoking. ${ }^{1,3,5,10}$

Green tea and bleeding after tooth extraction - Soltani et al conducted a randomized controlled clinical trial on 62 patients referred for mandibular molars extraction and were equally divided in to 2 groups and were given a green tea impregnated sterile gauze dressing in treatment group and non green tea containing gauze in control group and concluded that green tea extract contributes to significant decline in bleeding of socket after tooth extraction as well as reduction of oozing. ${ }^{16}$

\section{Modes of consumption of green tea}

1. As beverage

2. As mouth wash

3. As local drug delivery

4. As chewing gum ${ }^{10}$

Recommended dosage of green tea - Depending on brand, two or three cups of green tea per day (for a total of $240-320 \mathrm{mg}$ polyphenols) or $100-750 \mathrm{mg}$ per day of standardized green tea extract is recommended. ${ }^{5}$

\section{Side effects}

Although green tea is a beneficial natural product for health, its effects and constituents may be beneficial up to a certain dose only. But some unknown adverse effects may be caused with higher doses. Moreover, the effects of green tea catechins may differ from an individual to individual. The only negative side effect reported so far from drinking green tea is 'insomnia' because of its caffeine content. However, green tea contains less caffeine compared to coffee: there are, $30-60 \mathrm{mg}$ of caffeine in 6-8 ounces of tea, compared to over $100 \mathrm{mg}$ in 8 ounces of coffee. Patients sensitive to caffeine should be advised to use caffeine-free green tea or a caffeine-free extract to avoid restlessness, irritability, heart palpations, anorexia, stomach upset, nausea, frequent urination and skin rashes. Green tea contains vitamin $\mathrm{K}$ which may interfere with warfarin. In general, the level of fluoride in tea is inversely related to the EGCG contents, which means that the more natural EGCG 
in the tea leaves, the less is the fluoride content. Decaffeinated teas, when compared to their caffeinated counterparts, have an even higher fluoride content. According to Andreas Schuld of the Canadian 'Parents of Fluoride Poisoned Children', fluoride is considered a cancer promoter as it could possibly reduce the anti-cancer properties of tea or even cause cancer. The high fluoride content in green tea could also cause neurological and renal damage, especially in the presence of aluminium. In addition, the high fluoride content could cause osteoporosis, arthritis and other bone disorders. ${ }^{3}$ EGCG of green tea extract is cytotoxic and higher green tea consumption can exert acute cytotoxicity of liver cells. ${ }^{4}$ Due to higher tannin content green tea prevents iron absorption. So consuming several cups of green tea daily may not be good for iron deficient persons. ECGC binds to dihydrofolate reductase, so pregnant women consuming green tea in large amounts have risk of having child with spinia bifida and other neural tube defects. ${ }^{5}$ But based on the current literature, there does not appear to be any significant side effects or toxicity which may be caused due to regular green tea consumption. However patient should be aware of his general health and the side effects of the green tea before starting the green tea consumption. ${ }^{3,10}$

\section{Conclusion}

Green tea is consumed all over the world in various forms. The years of safe consumption of this beverage, supported by numerous studies showing health benefits, warrant a general recommendation to consume it on regular basis. Nowadays, green tea is being studied extensively worldwide as it is considered as one of the most promising dietary agents for the prevention and treatment of many diseases. Recent application of green tea in nanotechnology has suggested promising evidences in its bioavailability by delivering EGCG using lipid nanocapsules and liposome encapsulation. Further study in this regard can be useful in availing the healthy benefits of green tea. But further research on this field is still needed to define the actual magnitude of health benefits, so that the mechanisms of action can be elucidated, to establish the safe range of tea consumption associated with these benefits and to advocate for green tea for prevention and treatment of specific oral as well as health morbidities. The developing biomarkers for green tea consumption, as well as molecular markers for its biological effects, will facilitate future research in this area.

\section{References}

[1]. Khurshid Z, Zafar MS, Zohaib S, Najeeb S, Naseem M. Green tea (Camellia Sinensis): Chemistry and oral health. TODENTJ. 2016;10(Suppl-1):166-73

[2]. Parmar N, Rawat M, Vijay KJ. Camellia Sinensis (Green tea): A review. Global J Pharmacol. 2012;6(2):52-9

[3]. Thiyam B, Ravindra SV, Devi MP, Yeluri G, Gadiyar A. Green tea- A healthy sip. IJSS Case Reports \& Review. 2015;1(12):55-60

[4]. Baskar S. Role of green tea in dental problems. IJCRCPS. 2014;1(4):73-7

[5]. Nair V, Bandyopadhyay P, Kundu D. Green tea: A friendly oral beverage?. IDJSR. 2012-13;1(3):6-12

[6]. Rashika V. Healthy benefits of green tea - A review. AJPCR. 2013;1(4):203-6

[7]. Gupta DA, Bhaskar DJ, Gupta RK, Karim B, Jain A, Dalai DR. Green tea: A review on its natural anti-oxidant therapy and cariostatic benefits. IBSPR. 2014;2(1):8-12

[8]. Venkateswara B, Sirisha K, Chava VK. Green tea extract for periodontal health. J Indian Soc Periodontol. 2011;15(1);18-22

[9]. Anand J, Rai N, Kumar N, Pankaj G. Green tea: A magical herb with miraculous outcomes. IRJP. 2012;3(5):139-48

[10]. Grover HS, Deswal H, Bhardwaj A. Green tea asan alternative therapy in medicine and dentistry: A review. IAMJ. 2015;3(2):51621

[11]. Gadagi JS, Chava VK, Reddy VR. Green tea extract as a local drug therapy on periodontitis patients with diabetes mellitus: A randomized case-control study. JISP. 2013;17(2):198-203

[12]. Moghbel A, Farjzadeh A, Aghel N, Agheli H, Raise N. The effect of green tea on prevention of mouth bacteria infection, halitosis and plaque formation on teeth. IJT. 2011;5(14):502-15

[13]. Forouzanfar A, Arab HR, Shafaee H, Mokhtari MR, Golestani S. The effect of green tea mouthwash (Camellia Sinensis) on wound healing following periodontal crown lengthening surgery; adouble blind randomized controlled trial. OJST. 2012;2:369-72

[14]. Sharma M, Nagori K, Soni S, Swarnakar H, Vaishnav S, Nazir M et al. Phytochemical constituents and pharmacological profile of green tea: An overview

[15]. Balsaraf S, Chole R. Green tea: Its potential health implications and other benefits. IJNPND. 2016;5(2):46-9

[16]. Soltani R, Haghighat A, Fanaei M, Asghari G. Evaluation of the effect of green tea extract on the prevention of gingival bleeding after posterior mandibular teeth extraction: A randomized controlled trial. Evi Based Complement Alternat Med. 2014:1-4 